\title{
Desenvolvimento de um kit de reator de leito fixo de bagaço de cana-de-açúcar para uso didático
}

\section{Development of fixed bed reactor kit using sugarcane bagasse for didactic use}

Rafaelle Gomes Santiago ${ }^{1}$, Francisco Klebson Gomes dos Santos ${ }^{2, *}$, Ricardo Henrique de Lima Leite ${ }^{3}$, Edna Maria Mendes Aroucha ${ }^{4}$, Andarair Gomes dos Santos ${ }^{5}$

Resumo: É de fundamental importância o desenvolvimento de práticas de laboratório nos cursos de engenharia, porém os altos custos dos kits didáticos muitas vezes inviabilizam sua aquisição. No entanto, o presente trabalho teve como objetivo desenvolver um kit para aulas de cinética e reatores, utilizando materiais de fácil aquisição e com caráter ambiental, uma vez que o leito fixo é composto por bagaço de cana-de-açúcar. Para estudar a cinética, variou-se o volume de álcool etílico alimentado e a altura de leito fixo no reator, observando o comportamento do rendimento e da produtividade. Avaliou-se a influência da adição do álcool etílico e ácido acético na produção deste e avaliou-se a atividade operacional do recheio através do reuso do mesmo. A acidez do produto aumentou com o passar do tempo e após cerca de 12 horas manteve-se constante. $\mathrm{O}$ rendimento teve um aumento proporcional à diminuição do volume de alimentação e ao aumento da altura do leito fixo. Aplicando os valores obtidos em modelos de cinética de primeira e segunda ordem, concluiu-se que o modelo cinético de primeira ordem se ajustou melhor aos dados obtidos.

Palavras-chaves: Reator; Bagaço de cana-de-açúcar; Leito fixo.

Abstract: It is very important the development of laboratory practice in engineering courses, but the high cost of educational kits often hamper their acquisition. However, the present work aims to develop a kit for lessons kinetics and reactors, using easily obtainable materials and environmental character, since the fixed bed is made from sugar cane bagasse. To study the kinetics vary the volume of ethyl alcohol and fed to a fixed bed high in the reactor, observing the behavior of the yield and productivity. We evaluated the influence of the addition of ethyl alcohol and acetic acid in the production of and evaluated the operational activity of bagasse performing the reuse of the same. The acidity of the product increased with time and after about 12 hours remained constant. The yield increase was proportional to the decreased volume and increased height of the fixed bed. Applying the values obtained in models of chemical kinetics first and second order, it was concluded that the model of the first order kinetics are best fit to the data.

Key words: Reactor; Sugarcane bagasse; Fixed bed.

\footnotetext{
*Autor para correspondência

Recebido para publicação em 13/12/2014; aprovado em 21/06/2015

${ }^{1}$ Graduada em Engenharia Química - DACS/UFERSA, Campus Mossoró-RN, Av. Francisco Mota, 572 - Bairro Costa e Silva, CEP: 59.625-900. E-mail: rafaelle_g.s@ hotmail.com

${ }^{2}$ Eng. Químico, Dr. Eng. Química, Professor - DACS/UFERSA Campus Mossoró-RN, Av. Francisco Mota, 572 - Bairro Costa e Silva, CEP: 59.625-900. Email: klebson@ufersa.edu.br

${ }^{4}$ Eng. Químico, Dr. Eng. de Processos, Professor - DACS/UFERSA Campus Mossoró-RN, Av. Francisco Mota, 572 - Bairro Costa e Silva, CEP: 59.625-900. E-mail: ricardoleite@ufersa.edu.br

${ }^{5}$ Agrônoma, Dr ${ }^{\mathrm{a}}$ Agronomia, Professora - DACS/UFERSA Campus Mossoró-RN, Av. Francisco Mota, 572 - Bairro Costa e Silva, CEP: 59.625-900. E-mail: aroucha@ufersa.edu.br

${ }^{5}$ Eng $^{a}$ Química, Dra Eng. Química, Professora - DACS/UFERSA Campus Mossoró-RN, Av. Francisco Mota, 572 - Bairro Costa e Silva, CEP: 59.625-900. Email: andarair@ufersa.edu.br
} 


\section{INTRODUÇÃO}

A cana-de-açúcar apresenta-se como um dos principais produtos agrícolas do Brasil desde a época da sua colonização e, de acordo com Karnitz Junior (2007), uma grande demanda no mercado interno e externo por álcool provocou um aumento significativo na plantação da cana e, consequentemente na geração de bagaço da mesma. Este resíduo, atualmente é alvo de diversos estudos e encontra um vasto campo de utilização.

Os processos biológicos encontram-se cada vez mais explorados na atualidade, uma vez que é de extrema importância para o desenvolvimento de vários produtos (DUTTA, 2008). Por causa disso, diversos estudos vêm sendo desenvolvidos nessa área com o objetivo de diminuir os custos para a realização de processos, além de simplificá-los cada vez mais e os tornarem mais propícios à realização.

O estudo das enzimas tem imensa importância prática, inclusive na área da agricultura, da tecnologia de alimento e da engenharia química, além da medicina. Estas funcionam como catalisadores biológicos em diversos processos (DUTTA, 2008).

De acordo com um estudo realizado por Scheidt et al. em 2003, os processos fermentativos são limitados pela cinética do processo. O modelo cinético possibilita uma previsão do comportamento dos reagentes ou dos produtos e auxilia na busca de melhorias para aperfeiçoar o processo. Este pode ser descrito por uma equação que pode ter diversas formas (DUTTA, 2008).

Este trabalho trata da formação de vinagre de álcool o qual, de acordo com Schmidell et al. (2001), é o mais indicado para fins não alimentícios por conter menor quantidade de material orgânico e de resíduos, sendo este trabalho um estudo sobre o uso do bagaço como recheio no processo de fermentação acética.

Baseado no estudo de produção de ácido acético, o presente trabalho tem como objetivo desenvolver um reator didático em batelada utilizando cana-de-açúcar como leito fixo e, a partir daí, realizar um estudo cinético da formação de vinagre de etanol. Mais especificamente, este estudo deve analisar os efeitos na acidez do produto quando ocorrerem mudanças no volume da solução de etanol alimentado no reator e na variação da altura do leito do mesmo. Além destes parâmetros, objetiva-se estudar a reutilização do bagaço e, ainda, realizar um estudo da cinética aplicando modelos de primeira e segunda ordem para o mesmo.

\section{MATERIAL E MÉTODOS}

O reator foi construído com cano hidráulico de PVC para ser conduzido o processo de fermentação. Foi obtido um pedaço cilíndrico de cano com diâmetro de $10 \mathrm{~cm}$ e altura de $24 \mathrm{~cm}$, sendo, portanto, o volume total do reator de, aproximadamente 2 litros.

$\mathrm{O}$ cano foi fechado em uma das extremidades por uma tampa, também de PVC, para servir de base. A tampa foi aderida ao cano, sendo colada com silicone. Na base do reator foi inserida uma válvula para a retirada da solução para ser feita a análise de acidez.

Um esquema do reator de PVC confeccionado é mostrado na Figura 1.
É importante citar que foi colocada uma tela de proteção na válvula, internamente ao reator, para evitar sua obstrução com o bagaço da cana-de-açúcar. Outra tela foi colocada na parte superior do reator para servir de proteção contra possíveis insetos.

O bagaço da cana-de-açúcar foi recolhido de uma moenda no interior da cidade de Russas, no Ceará. O mesmo foi deixado para secar ao sol por um período de, aproximadamente, sete horas. Depois do período de secagem, o bagaço foi desfiado para, posteriormente, funcionar como um recheio (leito fixo) no reator.

Neste estudo, portanto não foram inoculadas bactérias, sendo utilizadas as bactérias nativas presentes no bagaço de cana-de-açúcar. Estas são do gênero acetobacter e convertem o álcool etílico em ácido acético.

Foi preparada uma solução de álcool etílico $10 \%$ v/v para cada batelada. Para isso fez-se uso de uma proveta de $100 \mathrm{~mL}$, de água destilada e do reagente álcool etílico absoluto PA.

Com o objetivo de avaliar a influência de alguns fatores como: volume de alimentação do reator, altura do leito e reutilização do recheio em uma segunda batelada foi realizado um planejamento experimental onde a resposta era $o$ rendimento.

A concentração de álcool etílico foi mantida constante em $10 \%$ v/v, pois Aquarone et al. (2001) afirma que $11 \%$ é a concentração máxima que as bactérias suportam. A temperatura foi mantida em $27{ }^{\circ} \mathrm{C}$, já que é a temperatura ideal para atividade do micro-organismo.

Para cada batelada realizada, foram recolhidas amostras de $1 \mathrm{~mL}$ do produto, em intervalos de duas horas durante dezesseis horas, para analisar a possível conversão do álcool etílico em ácido acético. Para isso foram realizadas titulações para cada amostra do produto do reator utilizando uma solução de hidróxido de sódio $(0,01 \mathrm{M})$, previamente padronizada com biftalato de potássio. Dessa forma foi possível obter a concentração de ácido com o passar do tempo.

O álcool etílico utilizado da DINÂMICA QUÍMICA CONTEMPORÂNEA, de pureza mínima de 99,6\%. O biftalato de potássio foi da VETEC QUÍMICA FINA, de pureza mínima 99,5\% e peso molecular 204,22. A fenolftaleína utilizada foi da DINÂMICA QUÍMICA CONTEMPORÂNEA. O hidróxido de sódio foi da DINÂMICA QUÍMICA CONTEMPORÂNEA, de pureza mínima $97 \%$ e peso molecular 40 .

Para cada estudo realizado foi gerado um gráfico concentração versus tempo para analisar a acidez do produto com o passar do tempo. Os gráficos foram plotados no software Excel 2010. Cada experimento estudado foi realizado em triplicata.

A partir dos gráficos obtidos, foram testados modelos de cinética de primeira ordem e de cinética de segunda ordem, utilizando, respectivamente as Equações 1 e 2.

$$
\begin{aligned}
& \frac{d C_{A}}{d t}=k C_{A} \\
& \frac{d C_{A}}{d t}=k C_{A}^{2}
\end{aligned}
$$


onde,

$\mathrm{C}_{\mathrm{A}}$ : concentração final de reagente;

$\mathrm{C}_{\mathrm{A} 0}$ : concentração inicial de reagente;

$\mathrm{k}$ : constante cinética

$\mathrm{t}$ : tempo

Foram utilizadas as Equações 3 e 4 para calcular, respectivamente, o rendimento e a produtividade de cada batelada realizada.

$$
\begin{gathered}
R=\frac{Q_{P}}{Q_{T}} \cdot 100 \\
P=\frac{Q_{P} \cdot 10}{t}
\end{gathered}
$$

onde,

$\mathrm{R}=$ rendimento

$\mathrm{P}=$ produtividade

$\mathrm{Q}_{\mathrm{P}}=$ quantidade de produto formado

$\mathrm{Q}_{\mathrm{T}}=$ quantidade de produto que deveria ter sido obtido $\mathrm{t}=$ tempo

$\mathrm{O}$ valor de $\mathrm{Q}_{\mathrm{T}}$ utilizado para os cálculos corresponde à acidez máxima que o vinagre pode conter, a qual corresponde ao valor de $4 \mathrm{~g} / 100 \mathrm{~mL}$.

Depois de calcular os valores de rendimento e produtividade para cada experimento, foram gerados gráficos de superfícies de resposta.

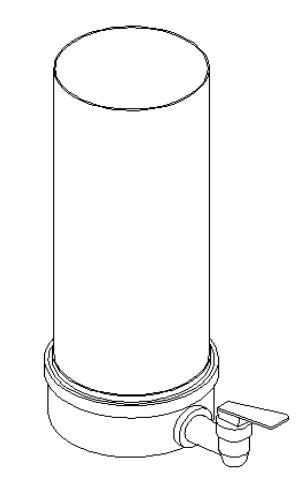

PERSPECTIVA

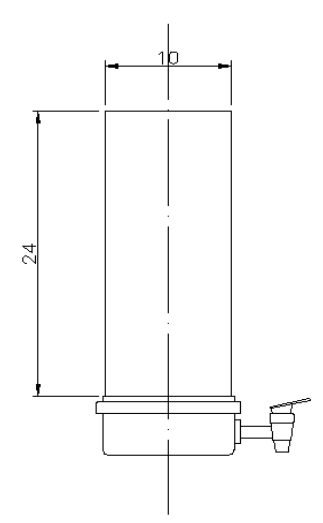

VISTA LATERAL

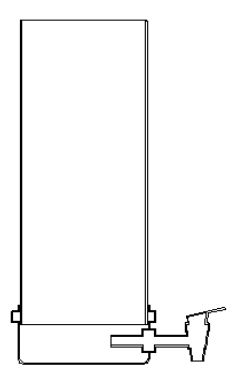

CORTE

Figura 1. Esquema do reator confeccionado. Mossoró, RN, 2015.

\section{RESULTADOS E DISCUSSÃO}

A Figura 2 mostra a evolução da acidez para três diferentes medidas do volume de solução alimentada no reator.

Através das curvas cinéticas geradas pelos diferentes volumes da alimentação no reator, percebe-se que todas se comportam da mesma maneira, ou seja, nas primeiras horas a acidez aumenta significativamente e, a partir de certo tempo tende a permanecer constante.

Resultados semelhantes quanto ao comportamento da acidez foram obtidos por Torres Neto et al. em 2005 ao estudar a produção de vinagre da fruta da palma da forrageira cujo processo obteve uma acidez constante após um período de 12 horas.

A maior produção de ácido é observada com o volume de $300 \mathrm{~mL}$, enquanto a menor é observada com $500 \mathrm{~mL}$. Alimentando o reator com $300 \mathrm{~mL}$ de álcool etílico, obtémse, em média, um produto com concentração de ácido de 2,16\%. Com $400 \mathrm{~mL}$, a acidez em média foi calculada em $2,01 \%$. Enquanto com $500 \mathrm{~mL}$, a acidez obtida com os processos foi, em média, 1,62\%. O mais provável é que, ao aumentar o volume de alimentação, a quantidade de microorganismos contidas no leito tenha sido insuficiente, de maneira que o produto obteve uma concentração de ácido menor.

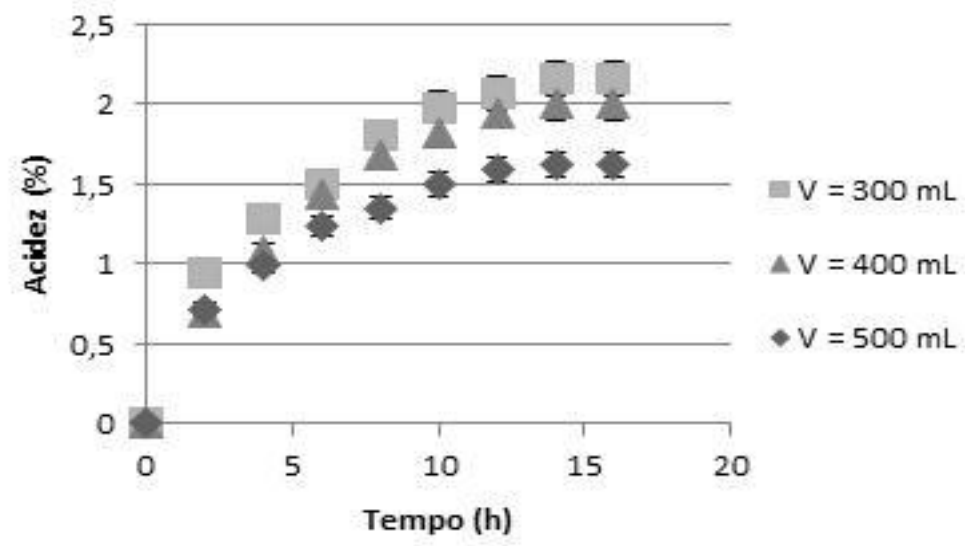

Figura 2. Curva de acidez para os volumes de etanol 300, 400 e $500 \mathrm{~mL}$ alimentados no reator, mantendo a altura do leito em 13 cm. Mossoró, RN, 2015. 
A Figura 3 mostra os resultados obtidos para um volume de $300 \mathrm{~mL}$, com alturas do leito variável. É fácil perceber que, ao aumentar a quantidade de recheio no reator, mantendo fixo o volume de solução de etanol, há um aumento na acidez do produto. Com seis centímetros de altura de leito fixo, a acidez do produto obtido foi, em média, 1,5\%. Com a altura do leito de $13 \mathrm{~cm}$, a acidez foi de 2,19\%. E com $20 \mathrm{~cm}$ de altura obteve-se uma acidez de $2,61 \%$.

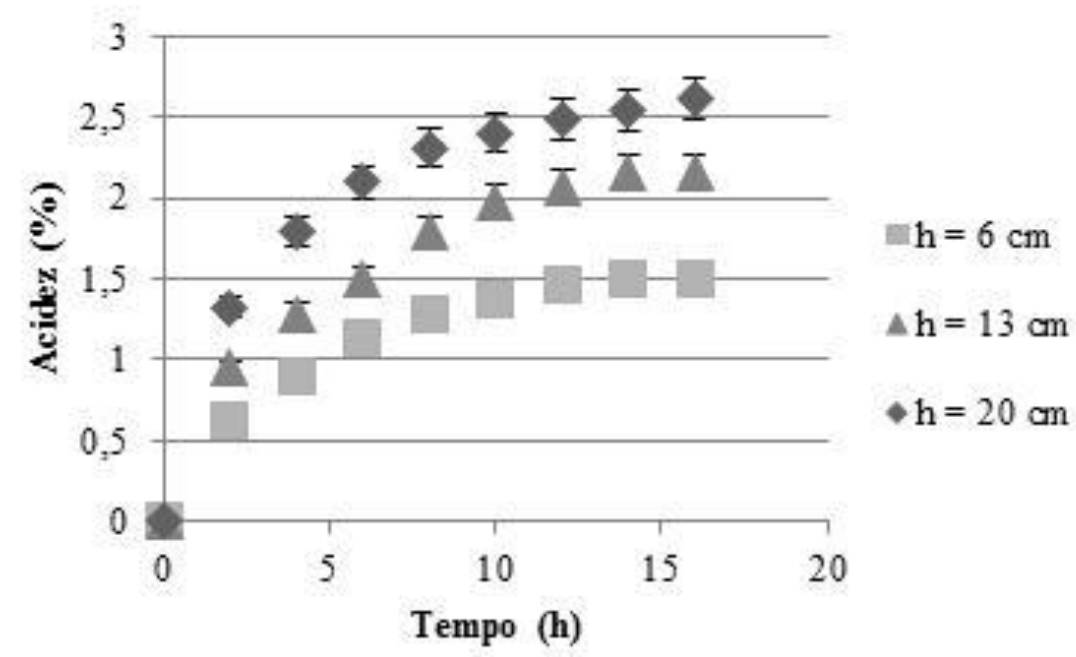

Figura 3. Curva de acidez para diferentes medidas de altura $(6,13$ e $20 \mathrm{~cm})$ com o volume de etanol de $300 \mathrm{~mL}$. Mossoró, RN, 2015.

Uma maior quantidade de microrganismos presentes no reator causa o aumento da conversão. No entanto, o bagaço de cana absorve uma grande quantidade de álcool etílico, sendo inviável a utilização de uma maior quantidade de bagaço como leito fixo.

No experimento realizado, embora com uma altura de 20 $\mathrm{cm}$ tenha sido obtida uma maior acidez, houve dificuldades de recolher alíquotas para análise. Foi considerado, portanto, que uma altura de $13 \mathrm{~cm}$ é mais conveniente para o processo.

A Figura 4 mostra a curva de acidez obtida fazendo uso do mesmo bagaço já utilizado em um processo nas mesmas condições de volume de alimentação de etanol e de altura do leito.

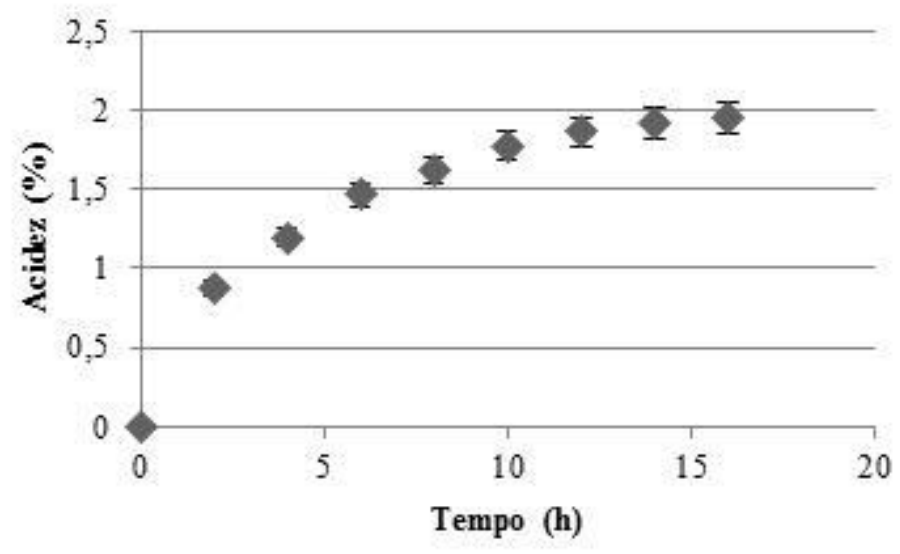

Figura 4. Curva de acidez para o experimento que reutiliza o bagaço do processo contendo um volume de $300 \mathrm{~mL}$ de etanol alimentado e $13 \mathrm{~cm}$ de altura do leito. Mossoró, RN, 2015.

Comparando a Figura 4 com a Figura 2, é possível concluir que o bagaço da cana-de-açúcar, mesmo reaproveitado, possui ainda micro-organismos suficientes para conversão do etanol em ácido acético. A acidez do produto possui concentração, em média, de 1,95\%, ou seja, a conversão de etanol em ácido acético fazendo uso do bagaço reaproveitado é apenas $10 \%$ menor da conversão obtida com o bagaço utilizado pela primeira vez no processo. Assim como nos casos anteriores, a curva de acidez considerando o reuso do leito também possui boa reprodutibilidade, observando que o desvio padrão é um valor muito pequeno.

$\mathrm{O}$ rendimento e a produtividade foram calculados para cada experimento e são mostrados na Tabela 1 
Tabela 1. Rendimento e produtividade para cada processo analisado.

\begin{tabular}{cccccc}
\hline Volume de álcool $(\mathrm{mL})$ & $\begin{array}{c}\text { Altura } \\
(\mathrm{cm})\end{array}$ & Rendimento $(\%)$ & $\begin{array}{c}\text { Tempo de } \\
\text { equilíbrio }(\mathrm{h})\end{array}$ & $\begin{array}{c}\text { Produtividade } \\
(\mathrm{g} / \mathrm{L} . \mathrm{h})\end{array}$ & $\begin{array}{c}\text { Produtividade total } \\
(\mathrm{g} / \mathrm{L})\end{array}$ \\
\hline 300 & 13 & 3,91 & 14 & 0,0926 & 1,2964 \\
400 & 13 & 3,01 & 14 & 0,0861 & 1,2054 \\
500 & 13 & 2,43 & 14 & 0,0694 & 0,9716 \\
300 & 6 & 2,25 & 14 & 0,0643 & 0,9002 \\
300 & 20 & 3,28 & 16 & 0,0978 & 1,5648 \\
300 (reutilização) & 13 & 3,91 & 16 & 0,0731 & 1,1696 \\
\hline
\end{tabular}

Considerando os valores de volume de álcool etílico e altura do leito, foram desenvolvidas superfícies de respostas para o rendimento e para a produtividade, uma vez que esses parâmetros são conhecidos (Tabela 1).
A Figura 5 mostra o rendimento de cada processo, considerando a extrapolação de valores dos três parâmetros.

A Figura 6 mostra a produtividade numa superfície de resposta ao volume e a altura do leito.

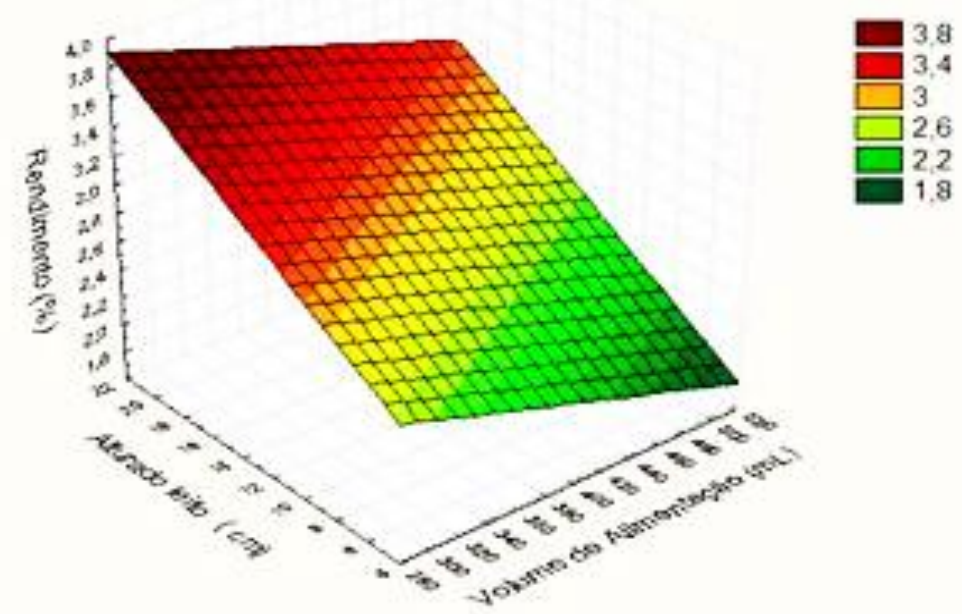

Figura 5. Resposta para o rendimento tendo como parâmetros o volume de etanol alimentado no reator e a altura do leito fixo. Mossoró, RN, 2015.

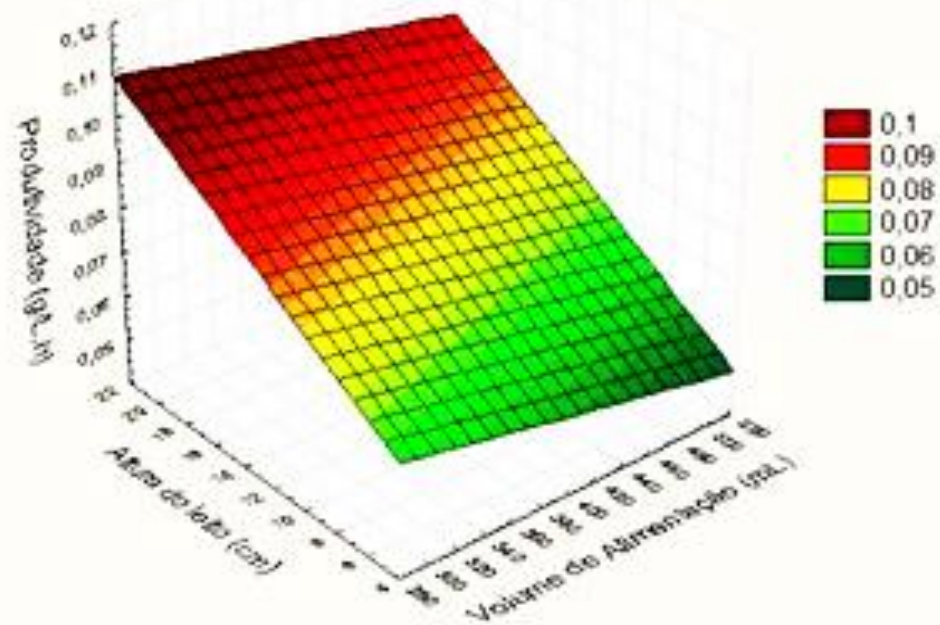

Figura 6. Resposta para produtividade tendo como parâmetros o volume de etanol alimentado no reator e a altura do leito fixo. Mossoró, RN, 2015. 
Além de obter as superfícies de respostas para o rendimento e a produtividade, é possível conhecer expressões teóricas para o cálculo desses dois parâmetros para quaisquer valores de volume e altura. Essas equações são mostradas nas Figuras 7 e 8.
Para conhecer o comportamento cinético da reação foram testados os modelos de primeira e de segunda ordens. Aplicando o modelo de primeira ordem, para o caso em que o reator é alimentado com $300 \mathrm{~mL}$ de álcool etílico com um leito fixo de altura $13 \mathrm{~cm}$, o gráfico obtém a forma mostrada na Figura 7.

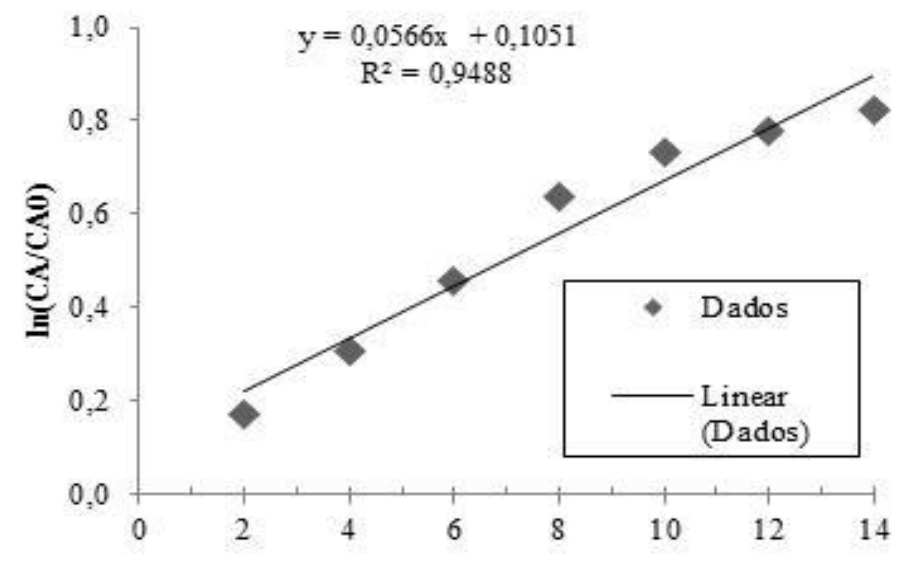

Tempo (h)

Figura 7. Aplicação do modelo cinético de primeira ordem para o caso em que o reator é alimentado com o volume de etanol de $300 \mathrm{~mL}$ e a altura do leito é $13 \mathrm{~cm}$. Mossoró, RN, 2015.

Analisando a Figura 7, percebe-se que os dados experimentais se ajustam bem ao modelo cinético de primeira ordem, embora alguns pontos encontrem-se dispersos. Isso foi possível concluir através do valor de coeficiente de correlação linear obtido igual a 0,9488. Ao aplicar o ajuste linear aos pontos, é obtida uma constante cinética no valor de $0,0566 \mathrm{~h}^{-1}$.

Este resultado é coerente com o experimento de Cechetti et al. (2011) cujos resultados também mostraram uma cinética de primeira ordem, porém com uma constante cinética mais elevada, nos valores de $0,4604 \mathrm{~h}^{-1}$ e $0,1929 \mathrm{~h}^{-1}$ para dois respectivos experimentos. Esse fato pode ser explicado pela metodologia aplicada por Cechetti et al. ser diferente, inclusive com a presença de um sistema de aeração contínuo, e como trata-se de um processo aeróbico, implica-se em uma maior conversão.

A Figura 8 mostra a aplicação ao modelo de segunda ordem ao gráfico de concentração versus tempo para o mesmo caso utilizado anteriormente.

Analisando a Figura 8 conclui-se que o processo não se ajusta ao modelo de segunda ordem, uma vez que não se comporta como uma reta após a aplicação do modelo em questão. $\mathrm{O}$ valor do coeficiente de correlação linear obtido $(0,835)$ mostra que os dados não se ajustam bem a esse modelo, ao contrário do modelo de primeira ordem $(0,9488)$.

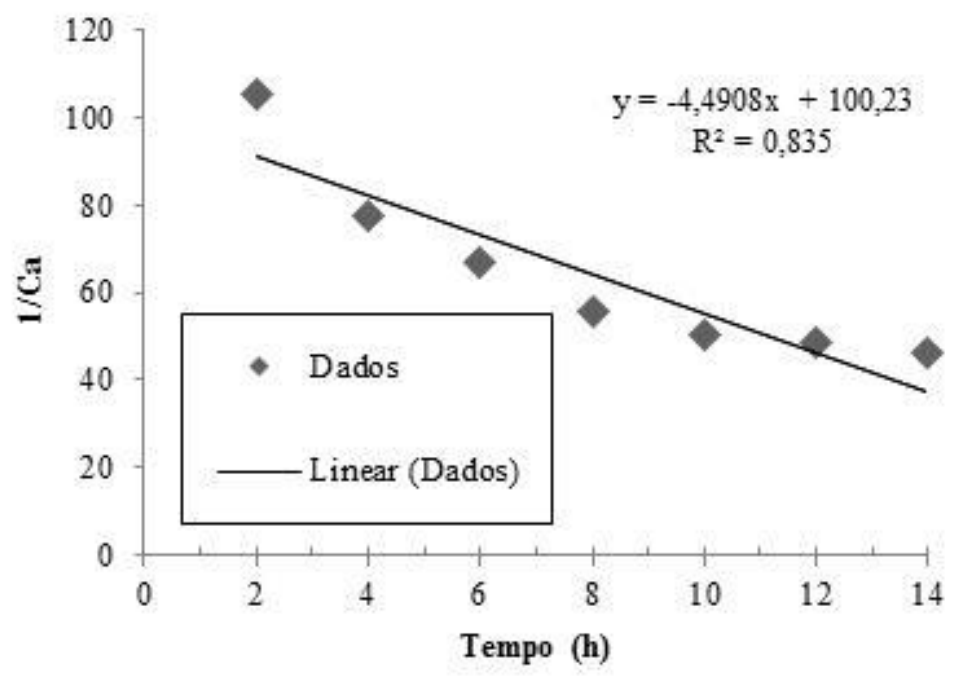

Figura 8. Aplicação do modelo de cinética de segunda ordem para o caso em que o reator é alimentado com o volume de etanol de $300 \mathrm{~mL}$ e a altura do leito é $13 \mathrm{~cm}$. Mossoró, RN, 2015. 


\section{CONCLUSÕES}

Com os experimentos realizados foi possível atingir o objetivo do trabalho quanto ao desenvolvimento de um reator de leito fixo para ser utilizado de forma didática. A aplicação do mesmo para a formação de vinagre de etanol ocorreu de forma satisfatória, observando que o volume de etanol utilizado e a quantidade de bagaço influenciam na acidez do produto e podem ser consideradas variáveis no processo. A melhor acidez foi gerada para o caso em que são alimentados $300 \mathrm{~mL}$ de etanol com uma altura do leito fixo em $13 \mathrm{~cm}$. Embora este caso não tenha obtido a melhor produtividade, o experimento foi mais conveniente no que diz respeito à retirada da amostra do reator para titulação e, consequente determinação do valor para acidez.

$\mathrm{O}$ baixo rendimento e a baixa produtividade demonstram que o processo deve ser melhorado para que haja uma maior conversão de álcool etílico em ácido acético. Para isso, poderia ser adicionado um sistema de aeração, uma vez que as bactérias do gênero presentes no bagaço da cana são aeróbicas.

Ao testar um modelo matemático, o modelo cinético de primeira ordem foi o que apresentou mais coerência com os resultados experimentais.

\section{REFERÊNCIAS BIBLIOGRÁFICAS}

ALCÂNTARA， S; SWARNAKAR, R. Influência de variáveis do processo de fermentação acética contínua sobre o rendimento. Artigo. Universidade Federal de Campina Grande, 2005.

AQUARONE, E.; LIMA, U.A.; BORZANI, W.; SCHMIDELL, W. Biotecnologia Industrial: Biotecnologia na produção de alimentos. Vol. 4. Editora Blücher, São Paulo, 2001.

CARVALHO, W.; SILVA, D.D.V.; CANILHA, L.; MANCILHA, I.M. Aditivos alimentares produzidos por via fermentativa. Parte I - Ácidos orgânicos. Artigo (Graduação em Engenharia Química) - Departamento de Biotecnologia, Faculdade de Engenharia Química de Lorena, 2005.

CECHETTI, R.; SCHEIDT, W.F.; SIPP, B.M.; BOMBARDELLI, C. Levantamento da Curva de Cinética de Primeira Ordem nos Processos de Fermentação Acética do Álcool. In: Encontro de Divulgação Científica e Tecnológica, 3., 2011, Toledo. Anais. Toledo: Universidade Federal do Paraná, 2011.

CORAZZA, Marcos L.; RODRIGUES, Dina G.; NOZAKI, Jorge. Preparação e caracterização do vinho de laranja. Química Nova, Maringá, n.4, p.449-452, 2001.
COSTA, C. M.; TAKAHASHI, J. S.; VILLAMONTE, M. R. Produção de vinagre. Universidade Federal de Santa Catarina. Departamento de Engenharia Química e Engenharia de Alimentos. 22 f. Florianópolis, 2006.

DUTTA, R. Fundamentals of Biochemical Engineering. Editora Springer, New Delhi, 2008.

KARNITZ JUNIOR, O. Modificação química do bagaço de cana e celulose usando anidrido do EDTA. Uso destes materiais na adsorção de metais pesados em solução aquosa. Ouro Preto, 2007. Dissertação (mestrado) Programa de Pós-Graduação em Recursos Hídricos. Universidade Federal de Ouro Preto.

MORETTO, E.; ALVES, R.F.; ARCHER, P.M.B.; CAMPOS, C.M.T.; PRUDÊNCIO, A.J. Vinhos e Vinagres (processamento e análises). Editora da UFSC. Florianópolis, 167p., 1988.

PEDROSO, P. M. F. Produção de vinagre de maçã em biorreator airlift. Dissertação de mestrado. FlorianópolisSC. Universidade Federal de Santa Catarina, 2003.

RIZZON, L.A. Sistema de Produção de Vinagre. EMBRAPA, 2006. Disponível em $<$ http://sistemasdeproducao.cnptia.embrapa.br/FontesHT ML/Vinagre/SistemaProducaoVinagre/fermentacao.htm> Acesso em 5 abr. 2006.

SCHEIDT, W.F.; CECHETTI, R.; PAPI, M.A.P.; ZANETTI, T.; BECKER, K.; BOMBARDELLI, C. Processo fermentativo rápido de produção de vinagre. Artigo. Universidade Tecnológica Federal do Paraná. Toledo, 2003.

SCHMIDELL, W.; LIMA, U.A.; AQUARONE,E.; BORZANI, W. Biotecnologia Industrial: Engenharia Bioquímica. Vol 2. Editora Blucher, São Paulo, 2001.

SEGATO, S.V.; PINTO, A.S.; JANDIROBA, E.; NÓBREGA, J.C.M. Atualização em Produção de Canade-Açúcar. Piracicaba, 2006.

SILVA, V.L.M.M.; GOMES, W.C.; ALSINA, O.L.S. Utilização do bagaço de cana de açúcar como biomassa adsorvente na adsorção de poluentes orgânicos. Artigo. Universidade Federal de Campina Grande, 2007.

TORRES NETO, A.B.; FEITOZA, A.C.O; SILVA, W.B.; SILVA, F.L.H. Estudo da produção de vinagre da fruta da palma forrageira por processo de fermentação batelada. In: Congresso Brasileiro de Engenharia Química em Iniciação Científica, 6, 2005, Campinas. Artigo: UNICAMP 